\title{
Vehicle Routing Algorithms to Intercept Escaping Targets
}

\author{
Pushkarini Agharkar ${ }^{1}$ and Francesco Bullo ${ }^{2}$
}

\begin{abstract}
We study a dynamic vehicle routing problem for moving targets. Our setup is as follows: (i) targets appear uniformly distributed on a unit disk via a temporal Poisson process and move radially outward with a constant speed $v$, (ii) a single vehicle with speed greater than that of the targets aims to intercepts them before they escape the disk. With the goal of maximizing the fraction of captured targets in the steady state, we propose three policies: Capturable Nearest Neighbor (CNN), Sector Wise (SW) and Stay Near Boundary (SNB) policy. We derive lower bounds on the fraction of targets captured by the CNN, SW and SNB policies. The CNN policy is shown to be optimal for arrival rate $\lambda$ below a critical value. For arrival rates above another critical value, the fraction of targets captured by the SW policy is more than that of the CNN policy. Finally, the SNB policy is within a constant factor of optimal in the limiting regime of $v \rightarrow 0^{+}$and $\lambda \rightarrow+\infty$. We present numerical simulations to illustrate our results.
\end{abstract}

\section{INTRODUCTION}

Vehicle motion planning arises in many important autonomous vehicle applications such as surveillance and perimeter defense. In this paper we consider a vehicle routing problem in which a vehicle must intercept targets before they escape a bounded environment which is a disk of unit radius. This problem setup is motivated by applications such as perimeter defense, wherein the demands could be visualized as moving targets trying to escape a region under surveillance by a vehicle.

Vehicle routing problems (VRP) are concerned with planning optimal vehicle routes for providing service to a given set of customers. The traveling salesperson problem (TSP) is a specific instance of the VRP and has been extended to include variations such as the deadline-TSP [2].

Recently, researchers have looked at the TSP with moving objects. In [6] the authors consider objects moving on straight lines and consider the case when the objects are slower than the vehicle and when the vehicle is constrained to move parallel to the horizontal or vertical axes. The same problem is studied in [7], but with arbitrary vehicle motion, and it is called the translational TSP. The authors of [7] propose a polynomial-time approximation scheme to catch all objects in minimum time. Other variations of the problem are studied in [8] and [1].

In the VRP, the routes are planned with complete information of the customers, and thus the optimization is

This material is based upon work supported in part by NSF Award 1035917 and in part by ARO Award W911NF-11-1-0092.

${ }^{1}$ Pushkarini Agharkar is with the Department of Mechanical Engineering, University of California Santa Barbara, CA 93106-5070, USA agharkar@umail.ucsb.edu

${ }^{2}$ Francesco Bullo is with Faculty of Mechanical Engineering, University of California Santa Barbara, CA 93106-5070, USA bullodengineering . ucsb.edu static, and typically combinatorial [15]. In contrast, in the dynamic vehicle routing (DVR) vehicles must plan paths through service demand locations that arrive sequentially over time. DVR problems have been reviewed in [5], [13] and [12]. In [4] and [14] DVR problems involving targets moving with a constant velocity are studied. In [4], a single vehicle intercepts the targets eventually. In [14], the vehicle is required to intercept the targets before they reach a deadline. [10] study a DVR problem in which demands disappear if not serviced within a time window.

In this paper, we introduce a DVR problem in which demands appear uniformly distributed on a disk and move radially outward until they escape the disk. The arrival process is Poisson with rate $\lambda$ and the demand speed $v \in$ $(0,1)$ is fixed. This problem is referred to as the Radially Escaping Targets (RET) problem for convenience. The goal of this paper is to design routing policies for a single vehicle in order to maximize the fraction of demands captured in the steady state.

The contributions of this paper can be summarized as follows. For any policy for the RET problem, we determine a policy independent upper bound on the fraction of targets captured. We also propose a method to establish upper and lower bounds on the path through radially escaping targets. Next, we formulate three policies: Capturable Nearest Neighbor (CNN), Sector Wise (SW) and Stay Near Boundary (SNB) policy. Lower bounds on the fraction of targets captured using the CNN, SW and SNB policies are obtained. The CNN policy is shown to be optimal for arrival rate below a critical value for all target speeds. For arrival rates above another critical value, the capture fraction of the SW policy is more than that of the CNN policy. The SW policy is thus more effective than the CNN policy in that regime. In the limit of low target speed and high arrival rate, the SNB policy is within a constant factor of optimal and the fraction of targets captured by the vehicle using the SNB policy asymptotically tends to 1 . We present numerical simulations which verify our results.

The paper is organized as follows. In Section II, we formally introduce the problem. In Section III, we present some background results. The CNN, SW and SNB policies are introduced in Section IV. Results from numerical simulations are presented in Section V.

\section{Problem Formulation}

We start with introducing a DVR problem in which targets appear independently and uniformly distributed in an environment $\mathscr{E}:=\{(r, \theta): 0 \leq r \leq 1 \forall \theta \in[0,2 \pi)\}$ with uniform spatial density and according to a temporal Poisson process 
with rate $\lambda$. Uniform spatial distribution of the targets is realized through probability density functions $f_{1}(r)=2 r$ and $f_{2}(\theta)=1 / 2 \pi$ where $r$ and $\theta$ are random variables describing the location of appearing targets in radial coordinates.

Once the targets appear, they move radially outwards with a constant speed $v \in(0,1)$ and eventually intercept the boundary of the environment. A vehicle with speed of 1 and confined to move in $\mathscr{E}$ intercepts the targets and captures them before they escape the environment. We refer to this problem as the Radially Escaping Targets (RET) problem for convenience. The parameters of the RET problem are the target speed $v$ and arrival rate $\lambda$.

Let the generation of targets commence at $t=0$. Let the vehicle start servicing the targets at $t=0$ as well. Let $n_{\text {capt }}(t)$ and $n_{\text {esc }}(t)$ be the number of targets that have been captured and have escaped respectively at time $t$. In this paper, the goal is to find policies which maximize the fraction of targets $c \in[0,1]$ captured by the vehicle where

$$
c:=\limsup _{t \rightarrow+\infty} \mathbb{E}\left[\frac{n_{\text {capt }}(t)}{n_{\text {capt }}(t)+n_{\text {esc }}(t)}\right]
$$

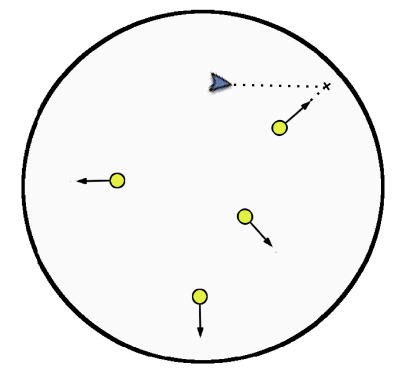

Fig. 1. Schematic of the Radially escaping targets (RET) problem

\section{PRELIMINARY RESULTS}

In this section, we provide some results which will be used to analyze policies for the RET problem.

\section{A. Constant bearing principle}

The optimal strategy (i.e. taking minimum time) for a vehicle to capture a target moving at a speed less than that of the vehicle is to move in a straight line with maximum speed to intercept the target based on the constant bearing principle[9]. Accordingly, the time taken by the vehicle starting from $(x, 0)$ to reach a demand located at $(r, \theta)$ and moving radially outward with constant speed $v$ is

$T(x, r, \theta)=\frac{-v(x \cos \theta-r)+\left(v^{2}(x \cos \theta-r)^{2}-\left(1-v^{2}\right)\left(2 r x \cos \theta-x^{2}-r^{2}\right)\right)^{1 / 2}}{1-v^{2}}$.

To distinguish static targets from moving targets, we introduce some terminology.

Definition 3.1: (Escaping target) A target moving radially outward is referred to as an escaping target.

A target is said to have been 'captured' by the vehicle if the vehicle reaches the target before it escapes the environment.



Fig. 2. Optimal vehicle location $x^{*}$ and the maximum probability $\rho^{*}$ of capturing an escaping target starting from $\left(x^{*}, 0\right)$ as a function of target speed $v$.

\section{B. Optimal placement of vehicle}

By optimal placement, we mean the location at which the vehicle should be placed in order for it to have the highest probability of capturing a target. To determine optimal placement, we start by defining the capturable set.

Definition 3.2: (Capturable set) A vehicle located at $(x, 0)$ can only reach demands located in the capturable set

$$
C(x, v):=\left\{(r, \theta): r<r_{c} \forall \theta \in[0,2 \pi)\right\}
$$

using the constant bearing principle, where

$$
r_{c}(x, v, \theta)=\max \left(0,1-v \sqrt{1+x^{2}-2 x \cos \theta}\right) .
$$

These are the locations for which $r+v T(x, r, \theta) \leq 1$. The expression for $r_{c}$ is obtained by setting $r_{c}+v T\left(x, r_{c}, \theta\right)=1$. The probability that a target is in the capturable set of a particular vehicle location $(x, 0)$ is

$$
\begin{aligned}
\rho(x, v) & :=\int_{0}^{2 \pi} \int_{0}^{1}[P(r, \theta) \in C(x, v)] f_{1}(r) f_{2}(\theta) d r d \theta \\
& =\int_{0}^{2 \pi} \int_{0}^{r_{c}} f_{1}(r) f_{2}(\theta) d r d \theta .
\end{aligned}
$$

When the vehicle is at location $\left(x^{*}(v), 0\right)$ where

$$
x^{*}(v):=\arg \max _{0 \leq x \leq 1} \rho(x, v),
$$

the probability of it capturing a target is maximum. The vehicle location $x^{*}$ is referred to as the optimal location. Let $\rho^{*}(v):=\rho\left(x^{*}, v\right)$. The variation of $x^{*}(v)$ and $\rho^{*}(v)$ is shown in Fig. 2. For target speed $v \leq 0.5, x^{*}=0$ and the vehicle location $(0,0)$ maximizes the probability of the vehicle being able to capture a target before it escapes. For higher speeds, this location is closer to the boundary. There is a qualitative difference between these two cases. In the former case, $(0,0)$ is the unique vehicle location which maximizes $\rho$ whereas for the later case, the set of corresponding optimal locations is $\left\{\left(x^{*}(v), \theta\right) \forall \theta \in[0,2 \pi)\right\}$.

We also define the expected time $T_{e}$ taken by a vehicle starting from $(x, 0)$ to service capturable targets appearing with a uniform spatial distribution in the environment.

$$
T_{e}(x, v):=\frac{\int_{0}^{2 \pi} \int_{0}^{r_{c}} T(x, r, \theta) f_{1}(r) f_{2}(\theta) d r d \theta}{\rho(x, v)} .
$$

Further, $T_{e}^{*}:=T_{e}\left(x^{*}, v\right)$. 


\section{Quantification of targets inside the environment}

The number of targets in an unserviced region in the environment is quantified now. We distinguish between targets originating and accumulating in a certain region. Targets are said to have accumulated in a region when after appearing, they spend time in the region in the course of their trajectories.

Lemma 3.3: (Expected number of targets) The expected number of targets at steady state in an unserviced

(i) disk of radius $s \leq 1$ centered at $(0,0)$ is $s^{3} \lambda / 3 v$ with probability density function $f_{a}(r)=\lambda r^{2} / v$ and

(ii) environment $\mathscr{E}$ is $\lambda / 3 v$.

Proof: Firstly, steady state is assumed, meaning that the initial transient has already passed, hence $t \geq 1 / v$. Also, by unserviced, we mean that the vehicle has not serviced targets in the region under consideration for at least time $1 / v$ before the time instant under consideration.

Let us examine the number of demands originating in the ring of radius $p \in(0,1]$ and accumulating in the annulus $\mathscr{B}_{r}$ of inner radius $r \in[p, 1-\Delta r]$ and thickness $\Delta r$. The intensity of the Poisson arrival process $\lambda_{p}$ on this ring is directly proportional to its area. Hence, $\lambda_{p}=2 p \lambda$. $P\left(\mathscr{B}_{r}\right.$ contains $n$ demands originating from $p)=P(n$ demands originated from $p$ in time interval $[t, t+\Delta r / v])=P(n$ demands originated from $p$ in time interval $\Delta r / v$ )

$$
=\frac{e^{\lambda_{p} \Delta r / v}\left(\frac{\lambda_{p} \Delta r}{v}\right)^{n}}{n !} .
$$

where $t=(r-p) / v$. Now, since Poisson processes are additive, the Poisson distribution of all the targets inside $\mathscr{B}_{r}$ has strength $\int_{p=0}^{r}\left(\lambda_{p} \Delta r / v\right) d p=\left(r^{2} \lambda \Delta r / v\right)$. It should be noted that the thickness $\Delta r \ll 1$ and the above expression does not include targets originating inside $\mathscr{B}_{r}$. Thus the expected number of targets in an unserviced annulus of inner radius $r$ and thickness $\Delta r$ due to targets originating from the disk of radius $r$ is $r^{2} \lambda \Delta r / v$.

Next, consider a disk of radius $s \leq 1$. Since Poisson processes are additive, the expected number of targets in this disk is the sum of expected number of targets inside disjoint annuli like $\mathscr{B}_{r}$. In integral form, the expected number of targets in the disk is $\int_{0}^{s}\left(r^{2} \lambda / v\right) d r=s^{3} \lambda / 3 v$. Let $f_{a}(r)$ be the probability density function of the accumulating targets in the disk, where $r$ denotes the random variable which is the radial coordinate of a target. Since $\int_{0}^{r} f_{a}(s) d s=r^{3} \lambda / 3 v$, we get $f_{a}(r)=\lambda r^{2} / v$.

\section{Bounds on paths and tours through static and escaping targets}

The following results are used to estimate and bound the length of the path/tour through targets in the environment.

Theorem 3.4: (Upper bound on path through escaping targets) Let targets starting from $\left(r_{i}, \theta_{i}\right), i \in\{1, \ldots, N\}$ move radially outward with speed $v$. Let $T$ be the length of the path through these escaping targets in some arbitrary order $\gamma:\{1, \ldots, N\} \rightarrow\{1, \ldots, N\}$. Let $T_{s}$ be the length of the path

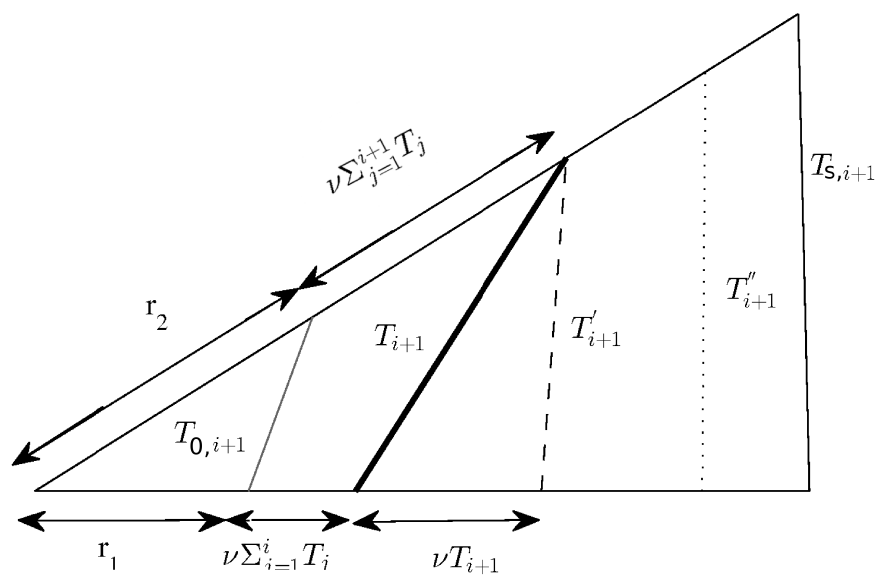

Fig. 3. The thick line labeled $T_{i+1}$ indicates the trajectory of the vehicle starting from the target $i$ to service the target $i+1$.

through static targets located at $\left(r_{i}+v \bar{T}, \theta_{i}\right), i \in\{1, \ldots, N\}$ processed in order $\gamma$ and $\bar{T} \geq T$. Then,

$$
T \leq \frac{T_{s}}{1-v} \text {. }
$$

Proof: Without loss of generality, let the targets be labeled in the order in which they are processed. Let the vehicle cover distance $T_{j}$ to service the $j$ th escaping target after having serviced the $(j-1)$ th escaping target. Consider the $i$ th escaping target starting from $\left(r_{i}, \theta_{i}\right)$. The vehicle services this target after having covered the distance $\sum_{j=1}^{i} T_{j}$. It then starts for the escaping target $i+1$ and reaches it in time $T_{i+1}$. Let $T_{i+1}^{\prime}$ be the distance between $\left(r_{i}+v \sum_{j=1}^{i+1} T_{j}, \theta_{i}\right)$ and $\left(r_{i+1}+v \sum_{j=1}^{i+1} T_{j}, \theta_{i+1}\right)$. Also, let $T_{i+1}^{\prime \prime}$ be the distance between $\left(r_{i}+v T, \theta_{i}\right)$ and $\left(r_{i+1}+v T, \theta_{i+1}\right)$ while $T_{s, i+1}$ is the distance between $\left(r_{i}+v T, \theta_{i}\right)$ and $\left(r_{i+1}+v T, \theta_{i+1}\right)$. Since the distance between two targets moving radially outward with the same speed is a non-decreasing function of time, $T_{i+1}^{\prime} \leq T_{i+1}^{\prime \prime} \leq T_{s, i+1}$. Referring to Fig. 3, from the triangle inequality, $T_{i+1}^{\prime}+v T_{i+1} \geq T_{i+1}$, i.e. $T_{i+1} \leq\left(T_{i+1}^{\prime}\right) /(1-v) \leq$ $\left(T_{s, i+1}\right)(1-v)$. Extending this to all the targets in the path,

$$
T=\sum_{i=1}^{n} T_{i+1} \leq \sum_{i=1}^{n} \frac{T_{s, i+1}}{1-v}=\frac{T_{s}}{1-v} .
$$

Theorem 3.5: (Lower bound on path through escaping targets) Let targets starting from $\left(r_{i}, \theta_{i}\right), i \in\{1, \ldots, N\}$ move radially outward with speed $v$. Let $T$ be the length of the path through these escaping targets in some arbitrary order $\gamma:\{1, \ldots, N\} \rightarrow\{1, \ldots, N\}$. Let $T_{0}$ be the length of the path through static targets located at $\left(r_{i}, \theta_{i}\right), i \in\{1, \ldots, N\}$ processed in order $\gamma$. Then,

$$
T \geq \frac{T_{0}}{1+v} .
$$

Proof: The proof is similar to that of Theorem 3.4 and is omitted for brevity.

Given a set $D$ of $n$ points, the Euclidean traveling salesperson problem (ETSP) is to determine the shortest tour, i.e., a closed path that visits each point exactly once. 


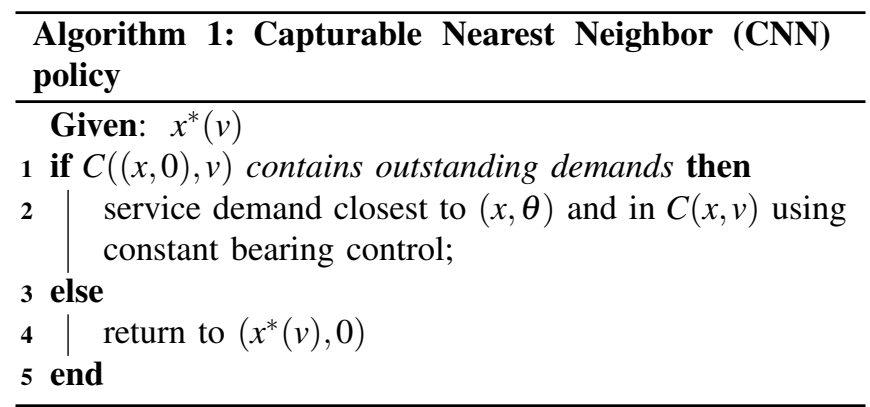

Theorem 3.6: (Asymptotic ETSP length,[3]) If a set D of $n$ points is distributed independently and identically in a compact set $Q$, then there exists a constant $\beta_{T S P, 2}$ such that

$$
\lim _{n \rightarrow+\infty} \frac{\mathbb{E}[\operatorname{ETSP}(D)]}{\sqrt{n}}=\beta_{T S P, 2} \int_{Q} \varphi(q)^{1 / 2} d q,
$$

where $\varphi$ is the density of the absolutely continuous part of the point distribution.

The constant $\beta_{T S P}$ has been estimated numerically as $\beta_{T S P} \approx$ $0.7120 \pm 0.0002[11]$.

\section{POLICIES}

In this section, we propose two policies for the RET problem. We start by providing an upper bound on the capture fraction $c$ for any policy for the RET problem.

Theorem 4.1: (Capture fraction upper bound) For every policy $\mathscr{P}$ for the $\operatorname{RET}(v, \lambda)$ problem, $c(\mathscr{P}) \leq \rho^{*}(v)$.

We now introduce two policies: the capturable nearest neighbor $(\mathrm{CNN})$ and the sector-wise (SW) policy.

\section{A. Capturable Nearest Neighbor (CNN) Policy}

At arrival rates below a critical value, optimizing the location at which the vehicle waits for new targets to arrive assumes importance. The CNN policy stated in Algorithm. 1 is motivated by this idea.

Theorem 4.2: (Performance of CNN policy) Given $\lambda_{c}=$ $1 /\left(2 \rho^{*} T_{e}^{*}\right)$, the expected fraction of targets $c$ serviced by the $\mathrm{CNN}$ policy satisfies

$$
c= \begin{cases}\rho^{*}(v), & \text { if } \lambda \leq \lambda_{c} \\ 1 /\left(2 \lambda T_{e}^{*}\right), & \text { if } \lambda \geq \lambda_{c}\end{cases}
$$

Theorem 4.3: (CNN policy lower bound) The expected fraction of targets $c$ serviced by the $\mathrm{CNN}$ policy satisfies the following conditions

$$
\begin{aligned}
& \text { if } x^{*}=0, c \geq \begin{cases}\rho^{*}(v) & \text { if } \lambda \leq 1 / 4 \\
1 / 4 \lambda\left(1+x^{*}\right), & \text { if } \lambda \geq 1 / 4\end{cases} \\
& \text { if } x^{*} \leq \frac{1-v}{v}, c \geq \begin{cases}\rho^{*}(v) & \text { if } \lambda \leq v / 4 \\
1 / 4 \lambda\left(1+x^{*}\right), & \text { if } \lambda \geq v / 4\end{cases} \\
& \text { if } x^{*} \geq \frac{1-v}{v}, c \geq \begin{cases}\rho^{*}(v) & \text { if } \lambda \leq v / 4 \\
v / 4 \lambda, & \text { if } \lambda \geq v / 4\end{cases}
\end{aligned}
$$

Remark 4.4: (Optimality of the CNN policy) When $\lambda \leq$ $\lambda_{c}$, the expected fraction of targets caught by the CNN policy from Theorem 4.2 is $\rho^{*}(v)$ and based on Theorem 4.1, this performance is optimal.

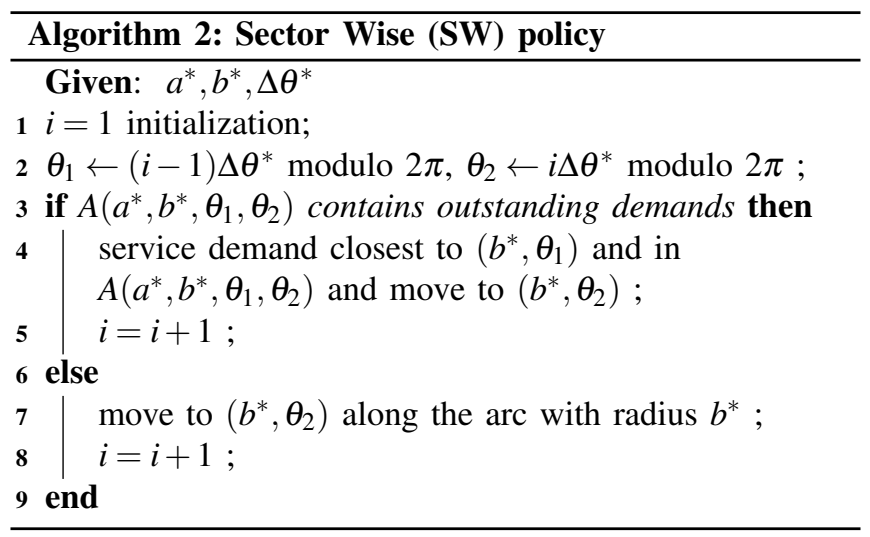

\section{B. Sector-wise policy (SW) Policy}

The SW policy stated in Algorithm. 2 is designed for the regime where $\lambda \geq 48 v / \pi(1-v)^{3}$. The vehicle traverses an annular sector in every iteration of the SW policy. The parameters $a^{*}, b^{*}$ and $\Delta \theta^{*}$ of the SW policy are optimized to maximize the capture fraction.

Definition 4.5: (Annular Sector) The set $A\left(a, b, \theta_{1}, \theta_{2}\right):=$ $\left\{(r, \theta): a \leq r \leq b \forall \theta \in\left[\theta_{1}, \theta_{2}\right]\right\}$, where $b \geq a \geq 0$.

Lemma 4.6: (Farthest capturable target) Given $A(a, b, 0, \Delta \theta)$ with $\Delta \theta \leq \pi$, if a vehicle traveling with unit speed and starting from $(b, 0)$ can capture a target located at $(b, \Delta \theta)$ and moving radially outward with speed $v$ then it can capture any target starting in $A(a, b, 0, \Delta \theta)$ and moving radially outward with speed $v$.

Theorem 4.7: (Routing in $A(a, b, 0, \Delta \theta)$ ) Given an unserviced annular sector $A(a, b, 0, \Delta \theta)$, with $0 \leq a<b<1$, the following holds true:

(i) If $a=\left(b^{3}-6 \pi v / \lambda \Delta \theta\right)^{1 / 3}$, then $\mathbb{E}[n]=1$ where $n$ is the number of outstanding targets in $A(a, b, 0, \Delta \theta)$.

(ii) If $\Delta \theta=\cos ^{-1}\left(\frac{b^{2}+1-(1-b)^{2} / v^{2}}{2 b}\right)$, then starting at $(b, 0)$, a vehicle can capture any moving target in $A(a, b, 0, \Delta \theta)$ before it escapes the disk.

(iii) If $2 \pi b \geq(b-a) / v$, then the vehicle starting from $(b, 0)$ at time $t_{0}$ and moving along the circle of radius $b$ will not return back to $A(a, b, 0, \Delta \theta)$ before all targets accumulated in $A(a, b, 0, \Delta \theta)$ at time $t_{0}$ escape $A(a, b, 0, \Delta \theta)$.

The constraints (i) - (iii) in Theorem 4.7 can be simplified and the maximum value $b^{*}$ that $b$ can take subject to these constraints can be found by solving the following concave maximization problem:

$$
\begin{array}{ll}
\text { maximize } & b \in\left[\frac{1-v \sqrt{2-v^{2}}}{2\left(1-v^{2}\right)}, 1\right) \\
\text { subject to } \quad b^{3} \Delta \theta & \geq \frac{12 \pi v}{\lambda}, \\
\Delta \theta & =\cos ^{-1}\left(\frac{b^{2}+1-(1-b)^{2} / v^{2}}{2 b}\right), \\
b^{3} \Delta \theta & \geq \frac{6 \pi v}{\lambda\left(6 \pi v-12 \pi^{2} v^{2}+8 \pi^{3} v^{3}\right)} .
\end{array}
$$


When $\lambda \geq 48 v / \pi(1-v)^{3}$, the concave maximization problem is feasible and can be solved to obtain $b^{*}$. The other two parameters can then be set:

$$
\begin{gathered}
\Delta \theta^{*}=\cos ^{-1}\left(\frac{b^{* 2}+1-\left(1-b^{*}\right)^{2} / v^{2}}{2 b^{*}}\right), \\
a^{*}=\left(b^{*}-\frac{12 \pi v}{\lambda \Delta \theta^{*}}\right)^{1 / 3} .
\end{gathered}
$$

Theorem 4.8: (SW policy lower bound) The expected fraction $c$ of targets serviced by the SW policy satisfies

$$
c \geq \frac{1}{\lambda\left(1-b^{*}+\sqrt{1+b^{* 2}-2 b^{*} \Delta \theta^{*}}\right)} .
$$

Theorem 4.9: ( $S W$ policy upper bound) The expected fraction $c$ of targets serviced by the SW policy satisfies

$$
c \leq \frac{1}{b^{*} \lambda \Delta \theta^{*}}
$$

\section{Stay Near Boundary (SNB) Policy}

The SNB policy is now introduced for the high arrival regime. In involves using solution to the Euclidean Minimum Hamiltonian Path (EMHP) problem which can be stated as follows:

Given a set $D$ of $n$ points, determine the length of the shortest path which visits each point exactly once.

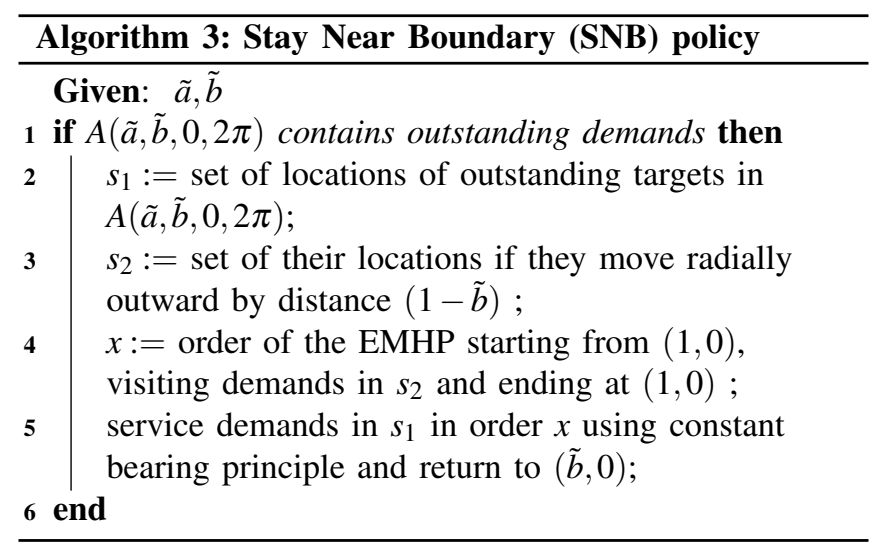

The parameters $\tilde{a}$ and $\tilde{b}$ of the SNB policy are solution of the following optimization problem:

$$
\begin{array}{cl}
\underset{a, b}{\operatorname{maximize}} & \left(\frac{1-v}{3 \beta_{T S P}}\right) \sqrt{\frac{2}{\lambda \pi v}}\left(\frac{b^{3}-a^{3}}{b^{2}-a^{2}}\right) \\
\text { subject to } & \delta_{1}\left(b^{2}-a^{2}\right) \leq(1-b), \\
& 1 \leq \delta_{2}(b+a), \\
& 3 v \leq\left(b^{3}-a^{3}\right), \\
& 0 \leq a<b<1 .
\end{array}
$$

where $\delta_{1}=k v /(1-v), \quad \delta_{2}=k v /(1+v)$ and $k=$ $\beta_{T S P} \sqrt{\lambda \pi / 2 v}$.
Theorem 4.10: (SNB policy lower bound) The expected fraction $c$ of targets serviced by the SNB policy as $\lambda \rightarrow+\infty$ satisfies

$$
c \geq \frac{(1-v)^{3}}{\beta_{T S P} \sqrt{2 \pi \lambda v}} .
$$

Proof: Consider the set $D:=\{(r, \theta) \in A(a, b, 0,2 \pi)\}$ of locations $(r, \theta)$ of targets accumulated in $A(a, b, 0,2 \pi)$. The normalized probability density function of the radial locations of these targets is $f(r)=3 r^{2} /\left(b^{3}-a^{3}\right)$ for $r \in[a, b]$. Let $\bar{D}$ be the set of locations $\left(r^{\prime}, \theta^{\prime}\right)$ of these targets if they move outwards by distance $d$ and occupy $A(a+d, b+d, 0,2 \pi)$. The normalized probability density functions for the random variables $r^{\prime}$ and $\theta^{\prime}$ which denote locations of these targets are

$$
\bar{f}\left(r^{\prime}\right)=\frac{3\left(r^{\prime}-d\right)^{2}}{b^{3}-a^{3}} \text { and } \bar{g}\left(\theta^{\prime}\right)=\frac{1}{2 \pi} .
$$

Using Theorem 3.6,

$$
\lim _{n \rightarrow+\infty} \frac{\operatorname{ETSP}(\bar{D})}{\sqrt{n}}=\beta_{T S P} \sqrt{\frac{3 \Delta \theta}{b^{3}-a^{3}}}\left(\frac{b^{2}-a^{2}}{2}\right) .
$$

Here $n=|D|$ and since the targets are accumulated in $A(a, b, 0,2 \pi), \mathbb{E}[n]=\lambda\left(b^{3}-a^{3}\right) / 3 v$. For $n \rightarrow+\infty$, the length of the ETSP through static targets $\bar{D}$ converges to the length of the EMHP starting from $s=(1,0)$, passes through each of the $n$ points in $D$ exactly once, and terminating at $f=(1,0)$. This follows from the fact that as $n \rightarrow+\infty$, the upper bound on the distance between two arbitrary points in $D$ is negligible when compared to the length of the tour/path. Using Theorem 3.4 and assuming that the vehicle traverses the path through moving targets in $D$ before they escape the environment, the expected length of the path through escaping targets accumulated in $A(a, b, 0,2 \pi)$ and located in the set $D$ is upper bounded by

$$
t_{u}:=\frac{\beta_{T S P}}{1-v} \sqrt{\frac{2 \pi \lambda}{v}}\left(\frac{b^{2}-a^{2}}{2}\right) .
$$

The condition

$$
\frac{\beta_{T S P}}{1-v} \sqrt{\frac{2 \pi \lambda}{v}}\left(\frac{b^{2}-a^{2}}{2}\right) \leq \frac{1-b}{v}
$$

ensures that the targets in $D$ are serviced before they escape the environment and Theorem 3.4 is applicable. The condition

$$
\frac{\beta_{T S P}}{1+v} \sqrt{\frac{2 \pi \lambda}{v}}\left(\frac{b^{2}-a^{2}}{2}\right) \geq \frac{b-a}{v}
$$

ensures that $A(a, b, 0,2 \pi)$ is unserviced when the vehicle begins its tour at $(1,0)$. Finally, when the condition $\left(b^{3}-\right.$ $\left.a^{3}\right) \geq 3 v$ is satisfied, $\mathbb{E}[n] \rightarrow+\infty$ as $\lambda \rightarrow+\infty$. The lower bound on the expected rate at which targets are serviced using the SNB policy is $\mathbb{E}[n] / t_{u}$ and

$$
c \geq\left(\frac{1-v}{3 \beta_{T S P}}\right) \sqrt{\frac{2}{\lambda \pi v}}\left(\frac{b^{3}-a^{3}}{b^{2}-a^{2}}\right) .
$$

Subject to the constraints in the optimization problem, $\left(b^{3}-\right.$ $\left.a^{3}\right) /\left(b^{2}-a^{2}\right) \geq 3(1-v)^{2} / 2$ and the result is obtained. 



Fig. 4. Performance of the (a) $\mathrm{CNN}$ and (b) $\mathrm{SW}$ policies for arrival rates $\lambda=2$ and $\lambda=10$ respectively.

Remark 4.11: (Necessary condition for SNB policy) A necessary condition for existence of a solution to the optimization problem in Theorem. 4.10 for the SNB policy is that $1 /$ delta $_{2}<2$ so that the second constraint in the problem leads to valid configurations $(a, b)$ for the policy. This is true when

$$
\lambda>\frac{(1+v)^{2}}{2 \pi \beta_{T S P}^{2} v} .
$$

Numerical simulations suggest that this condition is also sufficient for the existence of a solution to the optimization problem in Theorem 4.10.

Theorem 4.12: (SNB policy upper bound) The expected fraction $c$ of targets serviced by the SNB policy as $\lambda \rightarrow+\infty$ satisfies

$$
c \leq \frac{1+v}{\beta_{T S P} \sqrt{2 \pi \lambda v}} .
$$

Remark 4.13: (Optimality of $S N B$ policy) In the limit as $v \rightarrow 0^{+}$and $\lambda \rightarrow\left((1+v)^{2} 2 \pi \beta_{T S P}^{2} v\right)^{+}$, the capture fraction of the SNB policy satisfies $c \geq(1-v)^{3} /(1+v)$. Since $x^{*}=0$ and $\rho^{*}=(1-v)^{2}$ for $v \rightarrow 0^{+}$, the SNB policy is within a constant factor $(1-v) /(1+v)$ of optimal in this regime.

\section{Numerical Simulations}

We now present numerical simulations for the CNN and SW policies. The CNN policy gives optimal performance for $\lambda \leq \lambda_{c}$ based on numerical simulations. Its performance at $\lambda=2$ is examined. The actual simulation results, shown in Fig. 4(a) comply with the performance bounds obtained in Theorem 4.2 and 4.3. Performance of the SW policy in numerical simulations is shown for $\lambda=10$ in Fig 4(b).

Lastly, the lower bounds on the fraction of targets captured for both the CNN and SW policies are compared for range of parameters $v$ and $\lambda$ and the larger of the two values chosen, shown in Fig. 5. For a particular target speed, the CNN policy is suitable for lower arrival rates for the RET problem. The SW policy shows a better performance for higher arrival rates.

\section{CONCLUSION}

We have introduced a dynamic vehicle routing problem in which demands appear uniformly distributed on a disk according to temporal Poisson process. We propose three policies for a single vehicle to service the demands before they escape the disk. The CNN policy is optimal for arrival rate below a critical value for all target speeds $v \in(0,1)$. The performance of the SNB policy is close to optimal in

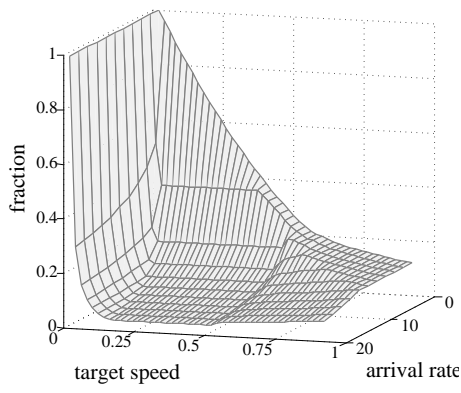

Fig. 5. Lower bound on $c$ from the CNN and SW policy is calculated numerically. The maximum of the two is shown for $\operatorname{RET}(v, \lambda)$.

the limiting regime of $\lambda \rightarrow+\infty$ and $v \rightarrow 0^{+}$. The SW policy is suitable for other regimes of the RET problem.

Extensions of the RET problem like for instance, when the distribution of targets in the environment is not uniform, or when the environment is an arbitrary closed curve are open to exploration.

\section{REFERENCES}

[1] Y. Asahiro, E. Miyano, and S. Shimoirisa. Grasp and delivery for moving objects on broken lines. Theory of Computing Systems, 42(3):289-305, 2008

[2] N. Bansal, A. Blum, S. Chawla, and A. Meyerson. Approximation algorithms for deadline-TSP and vehicle routing with time-windows. In Proceedings of the thirty-sixth annual ACM symposium on Theory of Computing, pages 166-174, 2004.

[3] J. Beardwood, J. Halton, and J. Hammersly. The shortest path through many points. In Proceedings of the Cambridge Philosophy Society, volume 55, pages 299-327, 1959.

[4] S. D. Bopardikar, S. L. Smith, F. Bullo, and J. P. Hespanha. Dynamic vehicle routing for translating demands: Stability analysis and receding-horizon policies. IEEE Transactions on Automatic Control, 55(11):2554-2569, 2010.

[5] F. Bullo, E. Frazzoli, M. Pavone, K. Savla, and S. L. Smith. Dynamic vehicle routing for robotic systems. Proceedings of the IEEE, 99(9):1482-1504, 2011.

[6] P. Chalasani and R. Motwani. Approximating capacitated routing and delivery problems. SIAM Journal on Computing, 28(6):2133-2149, 1999.

[7] M. Hammar and B. J. Nilsson. Approximation results for kinetic variants of TSP. Discrete and Computational Geometry, 27(4):635651, 2002.

[8] C. S. Helvig, G. Robins, and A. Zelikovsky. The moving-target traveling salesman problem. Journal of Algorithms, 49(1):153-174, 2003.

[9] R. Isaacs. Differential Games. Wiley, 1965.

[10] M. Pavone, N. Bisnik, E. Frazzoli, and V. Isler. A stochastic and dynamic vehicle routing problem with time windows and customer impatience. ACM/Springer Journal of Mobile Networks and Applications, 14(3):350-364, 2009.

[11] A. G. Percus and O. C. Martin. Finite size and dimensional dependence of the Euclidean traveling salesman problem. Physical Review Letters, 76(8):1188-1191, 1996.

[12] V. Pillac, M. Gendreau, C. Guéret, and A. L. Medaglia. A review of dynamic vehicle routing problems. European Journal of Operational Research, 225(1):1-11, 2012.

[13] H. N. Psaraftis. Dynamic vehicle routing: Status and prospects. Annals of Operations Research, 61:143-164, 1995.

[14] S. L. Smith, S. D. Bopardikar, and F. Bullo. A dynamic boundary guarding problem with translating demands. In IEEE Conf. on Decision and Control and Chinese Control Conference, pages 8543 8548, Shanghai, China, December 2009.

[15] P. Toth and D. Vigo, editors. The Vehicle Routing Problem. Monographs on Discrete Mathematics and Applications. SIAM, 2001. 